\title{
Deformation of cylindrical shells of steel 45 under complex loading
}

\author{
Stepan Cheremnykh", Vladimir Zubchaninov and Vadim Gultyaev
}

Tver state technical university, Naberezhnaya Afanasiya Nikitina, 22, Tver, Russia

\begin{abstract}
Reviewing the numerical simulation of the process of nonproportional elastic-plastic deformation of steel 45 by a knot of constant curvature, taking into account the complex nature of deformation under complex subcritical loading by axial compressive force and torque for a thin-walled circular cylindrical shell. The theory of Quas and simple processes of A. A. Ilyushin and the mathematical model of $\mathrm{V}$. Zubchaninov were applied taking into consider the parameters of the complex loading for plane trajectories To assess the accuracy of accepted theories, the simulation results are compared with experimental results, received on the automated complex $\mathrm{CN}$ - computer in the laboratory of the faculty of «Strength of materials and theory of elasticity and plasticity» of the Tver state technical University. Was introduced the scheme of calculations disproportionate plastic deformation of steel 45 using the proposed mathematical model showed a satisfactory result and recommended for further use. Remarks, that in the described processes the lack of some parameters complex loading in approximations reduces the accuracy of the final calculated values, differences significantly compared to the experimental data.
\end{abstract}

\section{Introduction}

The fundamental system-forming of the theory of processes elastic-plastic deformation of materials and basic equations are accepted by formulas $[1-8,10,11]$ :

$$
\left(\sigma_{i j}\right)=\sigma_{0}\left(\delta_{i j}\right)+\sigma\left(S_{i j}^{*}\right),\left(\varepsilon_{i j}\right)=\varepsilon_{0}\left(\delta_{i j}\right)+Э\left(Э_{i j}^{*}\right),(i, j=1,2,3),
$$

where

$$
\sigma_{0}=\frac{1}{3} \sigma_{i j} \delta_{i j}, \varepsilon_{0}=\frac{1}{3} \varepsilon_{i j} \delta_{i j}, \sigma=\sqrt{S_{i j} S_{i j}}, \ni=\sqrt{Э_{i j} Э_{i j}}
$$

- modules of ball tensors and deviator tensors respectively (first and second invariants);

$$
S_{i j}=\sigma_{i j}-\delta_{i j} \sigma_{0}, \ni_{i j}=\varepsilon_{i j}-\delta_{i j} \varepsilon_{0}, S_{i j}^{*}=\frac{S_{i j}}{\sigma}, \ni_{i j}^{*}=\frac{Э_{i j}}{Э}
$$

- the components of the diverters and their guide's tensors; $\delta_{i j}$ - the symbol of Kronecker.

\footnotetext{
* Corresponding author: cheremnykh_s.v@mail.ru
} 
With proportional (simple) loading of guide tensors stresses and strains matches, that $\left(S_{i j}^{*}\right)=\left(\Im_{i j}^{*}\right)$, and taking into account the elasticity of the volumetrically deformation have a place the relation of the theory of small elastic-plastic deformations of Ilyushin (4)

$$
\left(\sigma_{0}\right)=3 K \varepsilon_{0}, S_{i j}=\frac{\sigma}{\ni} \ni_{i j}=2 G_{p} \ni_{i j}, \sigma=\Phi(Э)(i, j=1,2,3),
$$

here $K$ - elastic modulus of bulk deformation; $2 G_{p}$ - doubled plastic shear modulus; $\sigma=\Phi(\ni)$ - the basic function of a unified deformation diagram of materials Roche's and Eichinger's, which for an arbitrary stress-strain state determines only the scalar properties of the material.

At disproportionate (complex) loading the guiding tensors of stresses and deformation, and also their speeds are not equal, so $\left(S_{i j}^{*}\right) \neq\left(\ni_{i j}^{*}\right),\left(\dot{S}_{i j}^{*}\right) \neq\left(\dot{\ni}_{i j}^{*}\right)$. In this case, the calculation in the defining relations (1) only scalar properties of materials is not enough. Ilyushin proposed $[1,2]$ a vector representation of deformations and stresses in a linear coordinate six-dimensional euclidean space with an orthonormal fixed basis $\hat{i}_{k}$, where $\mathrm{k}=0,1,2,5$. In this space stress and strain tensors are aligned with stress vectors $\bar{S}$ and strain $\bar{\varepsilon}$.

$$
\left\{\begin{array}{l}
\bar{S}=\bar{S}^{0}+\bar{\sigma}, \bar{S}^{0}=S_{0} \hat{i}_{0}, \bar{\sigma}=S_{k} \hat{i}_{k}, \\
\bar{\varepsilon}=\bar{\varepsilon}+\bar{\ni}, \bar{\varepsilon}=\ni_{0} \hat{i}_{0}, \bar{\ni}=Э_{k} \hat{i}_{k},
\end{array},(\mathrm{k}=1,2, \ldots, 5),\right.
$$

where $\bar{S}^{0}, \bar{\varepsilon}^{0}$ - stress and strain vectors of volumetric tension-compression in onedimensional space; $\bar{\sigma}, \bar{\ni}$ - the vectors of stress and strain forming in five-dimensional deviatory space $E_{5}$. Vector coordinates are associated with components of tensors and deviators by one-to-one transformations [1-3]

$$
\begin{aligned}
& S_{0}=\sqrt{3} \sigma_{0}, S_{1}=\sqrt{\frac{3}{2}} S_{11}, S_{2}=\frac{S_{22}-S_{33}}{\sqrt{2}}, S_{3}=\sqrt{2} S_{12}, S_{4}=\sqrt{2} S_{23}, S_{5}=\sqrt{2} S_{13}, \\
& Э_{0}=\sqrt{3} \varepsilon_{0}, Э_{1}=\sqrt{\frac{3}{2}} Э_{11}, Э_{2}=\frac{Э_{22}-Э_{33}}{\sqrt{2}}, Э_{3}=\sqrt{2} Э_{12}, Э_{4}=\sqrt{2} Э_{23}, Э_{5}=\sqrt{2} Э_{13},
\end{aligned}
$$

and the modules of the vectors $\bar{\sigma}$ and $\bar{\ni}$ relatively equal

$$
\sigma=\sqrt{S_{i j} S_{i j}}=\sqrt{S_{k} S_{k}}, \ni=\sqrt{Э_{i j} Э_{i j}}=\sqrt{Э_{k} Э_{k}} \text {. }
$$

In space $E_{5}$ the end of the vector of deformations $\bar{\ni}=Э \hat{\ni}$ describes the deformation path $\bar{\ni}(s)$ with the length of the arc $s$, which displays in this space the process of deformation of the material during its forming. At each point of this trajectory $\bar{\ni}(s)$ built natural generalized orthonormal riper Fresnes-Ilyushin $\left\{\hat{p}_{k}\right\}, \mathrm{k}=1,2, \ldots, 5$, and also the stress vector $\bar{\sigma}=\sigma \hat{\sigma}$ and its incrementd $d \bar{\sigma} / d s$, where $\hat{\vartheta}$ and $\hat{\sigma}$ - unit strain and stress vectors, subsequently. The aggregate of the trajectory deformation $\bar{\ni}(s)$ and the vectors $\bar{\sigma}, d \bar{\sigma} / d s$ constructed at each of its points along with the corresponding temperature $\mathrm{T}$, by the module of $\varepsilon_{0}$ and nonthermomechanical parameters $\beta$ form a geometric image of the physical process of deformation and deviator space $E_{5}$. It is obvious that the direction and length of vectors $\bar{\sigma}$ and $\bar{\ni}$, it is obvious that the direction and length of vectors, will 
depend on invariants of tensors, parameters of curvature and torsion of the trajectory $k_{m}$ $(\mathrm{m}=1,2,3,4)$, temperatures $\mathrm{T}$ and parameters $\beta$.

Based on the particular postulate of isotropy A. A. Ilyushin [1], V. G. Zubchaninov received [3] defining relations between stress and strain vectors in $E_{5}$, the local form of which for threedimensional problems has the form

$$
\frac{d \bar{\sigma}}{d s}=M_{1} \hat{p}_{1}+M \hat{\sigma}+M_{3} \hat{p}_{3}
$$

here

$$
\hat{\sigma}=\frac{\bar{\sigma}}{\sigma}=\cos \vartheta_{1} \hat{p}_{1}+\sin \vartheta_{1}\left(\cos \vartheta_{2} \hat{p}_{2}+\sin \vartheta_{2} \hat{p}_{3}\right)
$$

unit stress vector; $\vartheta_{1}, \vartheta_{2}$ - angles of approach and deplanation, which are the polar spherical coordinates of the vector $\bar{\sigma}$ in the movable frame Fresnes $\left\{\hat{p}_{k}\right\},(\mathrm{k}=1,2,3)$, for which

$$
\begin{gathered}
\hat{p}_{1}=\frac{d \bar{\Im}}{d s}, \hat{p}_{2}=\frac{1}{k_{1}} \frac{d^{2} \bar{\Im}}{d s^{2}}, \hat{p}_{3}=\frac{1}{k_{2}}\left[k_{1} \frac{d \bar{\Im}}{d s}+\frac{d}{d s}\left(\frac{d^{2} \bar{\Im}}{d s^{2}}\right)\right] ; \\
M=\frac{d \sigma}{d s}-M_{1} \cos \vartheta_{1}-M_{3} \sin \vartheta_{1} \sin \vartheta_{2} ;
\end{gathered}
$$

$M_{1}, M_{3}, d \sigma / d s$-functional deformation process, dependent from the parameters of the complexity of the process: $s$ - arc lengths of the deformationpath, angles of its fracture $\vartheta_{1}^{0}$ and curvature parameters $k_{1}$ and torsion $k_{2}$.

To determine angles $\vartheta_{1}$ and $\vartheta_{2}$ we need to use differential nonlinear equations [3]

$$
\begin{aligned}
& \frac{d \vartheta_{1}}{d s}+k_{1} \cos \vartheta_{2}=\frac{1}{\sigma}\left(-M_{1} \sin \vartheta_{1}+M_{3} \cos \vartheta_{1} \sin \vartheta_{2}\right), \\
& \sin \vartheta_{1}\left(\frac{d \vartheta_{2}}{d s}+k_{2}\right)=k_{1} \cos \vartheta_{1} \sin \vartheta_{2}+\frac{M_{3}}{\sigma} \cos \vartheta_{2} .
\end{aligned}
$$

In the case of flat trajectories at $\vartheta_{2}=0, k_{2}=0$ from (8), (12) we get

$$
\frac{d \bar{\sigma}}{d s}=M_{1} \frac{d \bar{\Im}}{d s}+\left(\frac{d \sigma}{d s}-M_{1} \cos \vartheta_{1}\right) \frac{\bar{\sigma}}{\sigma}, \frac{d \vartheta_{1}}{d s}=-\left(\frac{M_{1}}{\sigma} \sin \vartheta_{1}+k_{1}\right) .
$$

Angle of approach $\vartheta_{1}$, which characterizes the deviation of the stress vector $\bar{\sigma}$ from the tangent to the trajectory of the deformation at each point is a functional of the parameters of the complexity of the process $\vartheta_{1}=\vartheta_{1}\left(s, \vartheta_{1}^{0}, k_{1}\right)$. This angle reflects the effect of the vector properties of the material on the deformation process, a $\sigma=\sigma\left(s, \vartheta_{1}^{0}, k_{1}\right)$, being a functional of the same parameters - the influence of scalar properties of the material.

In simple cases of complex loading, close to simple, mean that, when the deformation trajectories are close to the linear proportional loading, for example, the trajectories of small curvature, the approximate law of Odqvist-Ilyushin can be used

$$
\sigma=\Phi(s)
$$

To smellier to the universal law of the Rosh, and Eichinger $\sigma=\Phi(Э)$ in case of simple loading. In (14) always $s \geq \ni$ and in an obvious form does not take into account the history of complex loading, what mean that we take in account, that it has little effect on the processes of complex loading. Such neglect of parameters $k_{1}$ and $\vartheta_{1}^{0}$ in the interrelation 
(14) in practical calculations, it can lead to unreliable results, especially for the trajectories of medium and large curvature, as well as trajectories with large angles of fracture $\vartheta_{1}^{0} \geq 90^{\circ}$. Therefore, the creation of new mathematical models, describing inelastic deformation of materials and construction on their basis of more accurate approximations of functional, taking into account all the parameters of the complexity of the process, is an important task of the theory of plasticity.

\section{Mathematical model of the theory of processes in flat tasks.}

The main equations of the mathematical model of the theory of processes in flat tasks are the defining relations (13) and universal approximations of functional V. G. Zubchaninov $[3,9]$

$$
\begin{gathered}
\sigma=\sigma\left(s, \vartheta_{1}^{0}, k_{1}\right)=\Phi(s)+A f_{0}^{p} \Omega(\Delta s)-B \Delta s k_{1}, \\
\frac{d \sigma}{d s}=\frac{d \Phi}{d s}+A f_{0}^{p} \frac{d \Omega}{d s}-B \frac{d}{d s}\left(\Delta s k_{1}\right), \\
M_{1}=2 G_{p}+\left(2 G-2 G_{p}^{0}\right) f^{q},
\end{gathered}
$$

where $\Delta s=s-s_{K}^{T}$ - the increment of the arc of the trajectory deformation; $s_{K}^{T}$ - the length of the arc at the point of its fracture; $G, G_{p}$ - elastic and plastic shear modulus for initial isotropic material; - $G_{p}^{0}$ value $G_{p}$ at the culminating point of;

$$
\Omega(\Delta s)=-\left(\gamma \Delta s e^{-\gamma \Delta s}+b\left(1-e^{-\gamma \Delta s}\right)\right),
$$

- function describing the «dive» of the module of the vector of the generalized stresses and the effect of baushinger at the complex unloading and subsequent plastic deformation;

$$
f=f\left(\vartheta_{1}\right)=\frac{1-\cos \vartheta_{1}}{2}, f_{0}=f\left(\vartheta_{1}^{0}\right)=\frac{1-\cos \vartheta_{1}^{0}}{2}
$$

- function of complex loading, that taking into account the orientation of the stress vector in the deformation process and its value at the break point of the trajectory; $A, B, b, \gamma, p, q$ material parameters for each construction material, experimentally determined from basic experiments. Generalized to complex loading, the effect of baushinger regarded as a manifestation of General properties of delay scalar properties of materials [3].

Under given initial conditions with specified functional (15)-(16), constitutive relation (13) leads to Cauchy problem, which was solved using the fourth-order numerical RungeKutta method, The solutions obtained by comparing the calculated and experimental data allow the verification of different versions of the model, including when some parameters of the complexity of the process are not taken into account in the approximation of the functionals.

\section{Results of the performed experiments and numerical simulation}

The experimental results were obtained on the automated complex SN-computer in the laboratory of the faculty of "strength of materials, theory of elasticity and plasticity» Tver state technical University [4]. The experiments were carried out on thin-walled circular cylindrical shells made of steel 45 . The studies were carried out at elastic-plastic deformation (hard loading) thin-walled tubular sample along a flat trajectory, containing two straight sections and a section of a circle of constant curvature. 
Tubular samples made of steel 45 with mechanical characteristics $E=2,05 \mathrm{MPa}$, $\mu=0,3, G=0,788 \mathrm{MPa}$, wall thickness $h=1 \mathrm{~mm}$, radius of the middle surface $r=15,5 \mathrm{~mm}$, the length of the working part $l=110 \mathrm{~mm}$, shown in figure 1.

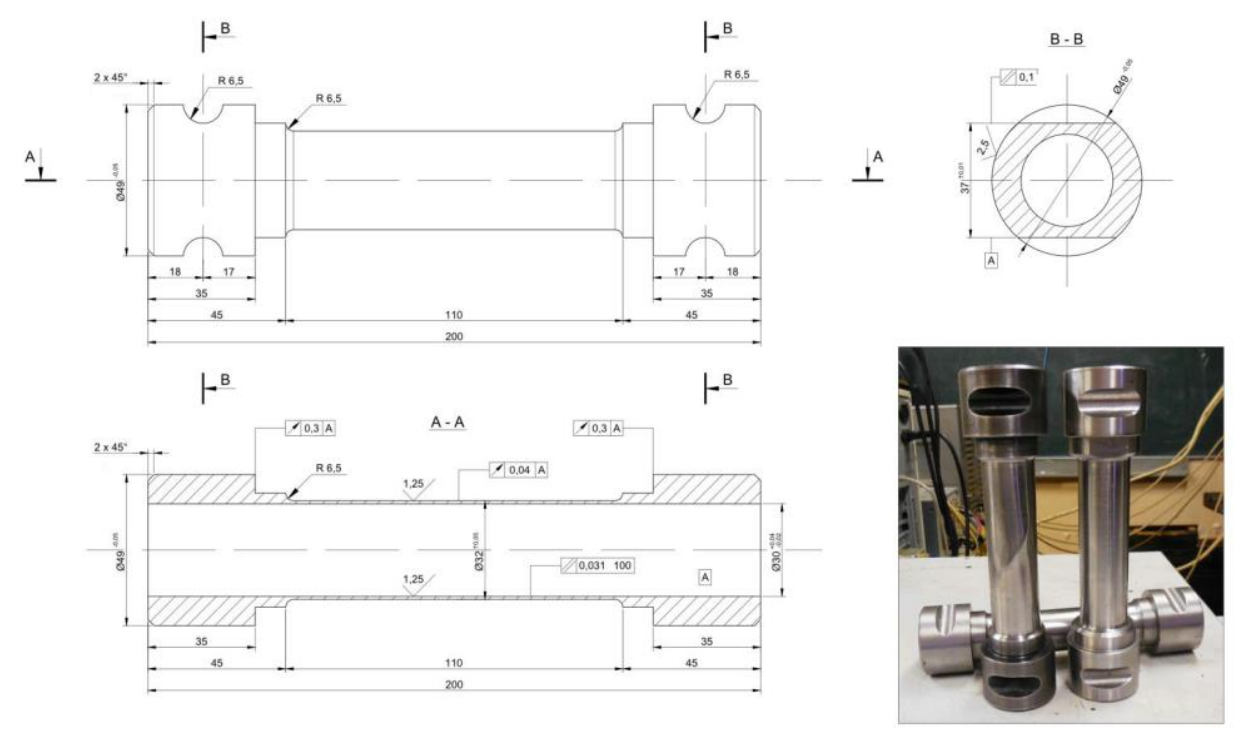

Fig. 1. Examination sample

To check the initial isotropy of the material, a comparison of deformation diagrams is performed $\sigma=\Phi(Э)$ simple loading processes for tensile specimens, torsion and internal pressure, which are shown in the figure 2.

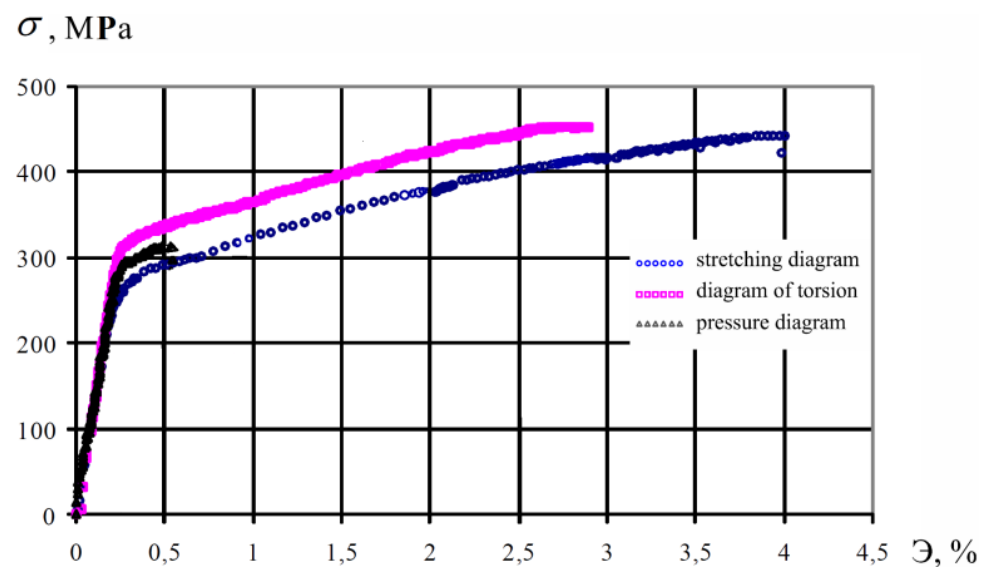

Fig. 2. Diagrams of material deformation under simple loading processes

Comparison of deformation diagrams allowed us to conclude that, the material of specimens is conventionally isotropic, as in the developed plastic deformation ranges of values of the module of the vector of stresses does not exceed $10 \%$.

Test programs of complex loading under normal temperature conditions under disproportionate influence of axial force and torque were carried out at a constant speed $\dot{\varepsilon}=10^{-6} \sec ^{-1}$ in a plane $Э_{1}-Э_{3}$ deviator space of deformations $E_{5}$. In a series of tests 
carried out, there are multiple trajectories of deformation with sections of different constant curvature $\kappa_{1}=$ const $[12,13]$. One of these three-link trajectories is shown in the figure 3.

$\ni_{1}, \%$

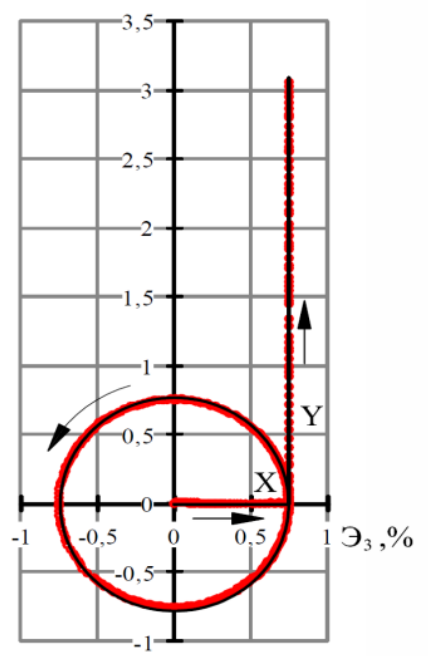

Fig. 3. Stress-strain diagram of the material under complex loading processes

On the first straight section torsion to the value was realized $\ni_{3}=0,75 \%$. In point $X$ the second section begins, where the angle fracture is $\vartheta_{1}^{0}=90^{\circ}$ and carried out one round of non-proportional tension with torsion of the shell in the form of the circumferential path of radius $R=\ni=0,75 \%$ and curvature $\kappa_{1}=100$. At the point $\mathrm{Y}$ originates the third section, where the stretching to the loss of stability while maintaining a constant level of tensional deformation $\ni_{1}$ on the last link.

Figure 4 shows the response to the implemented deformation trajectory in the plane deviator stress space

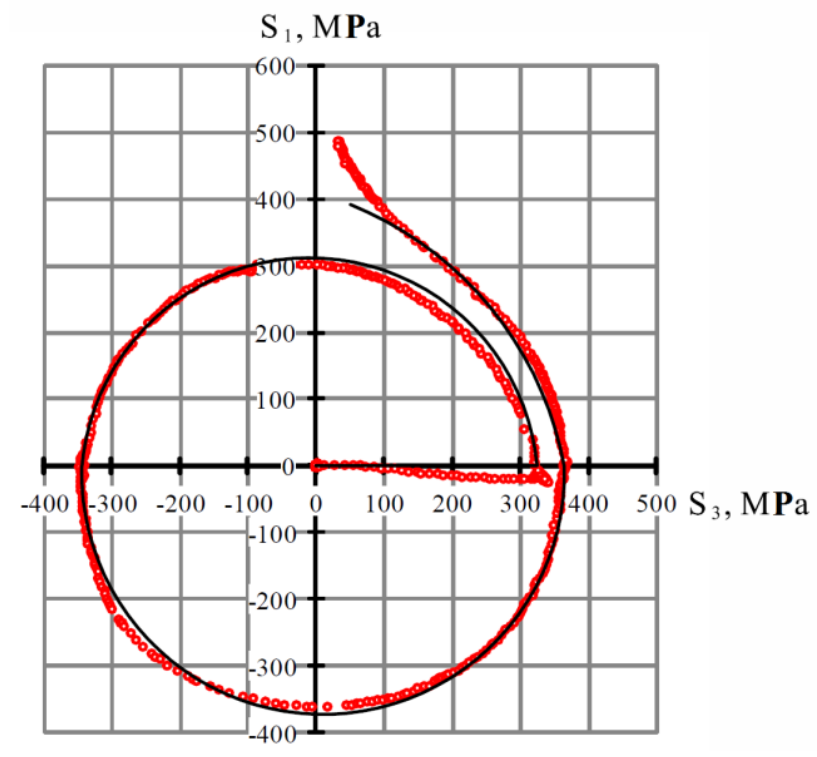

Fig. 4. Response to the realized deformation trajectory in the plane $S_{1}-S_{3}$ 
Figure 5 shows the diagram $\sigma-s$, characterizing the scalar properties of the material

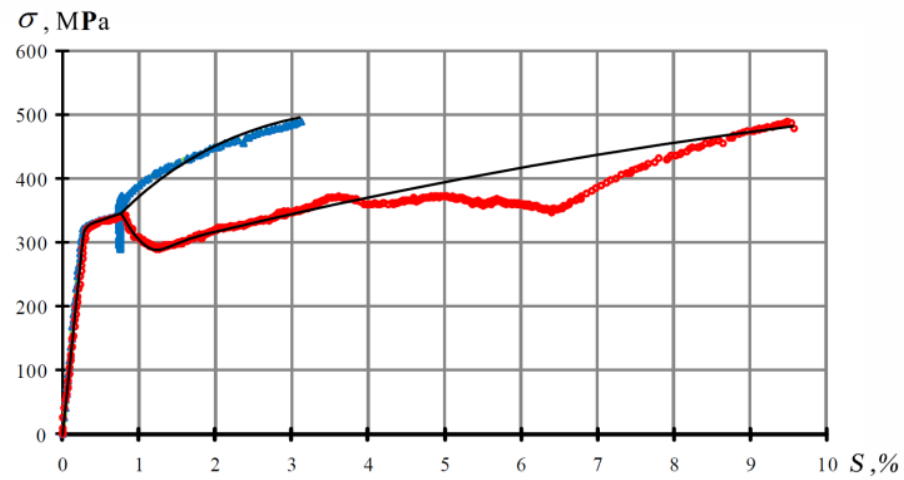

Fig. 5. Scalar material properties

Figure 6 shows the $\vartheta_{1}-\sqsupset s$ diagram describing the vector properties of the material $\vartheta_{1}, \mathrm{rad}$

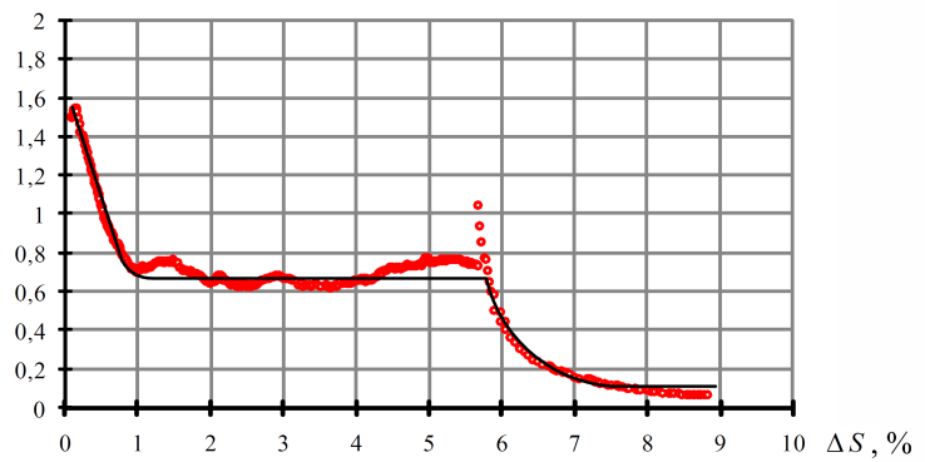

Fig. 6. Vector material properties

Figure 7 shows the local deformation diagram of the material tension-compression $S_{1}-\ni_{1}$

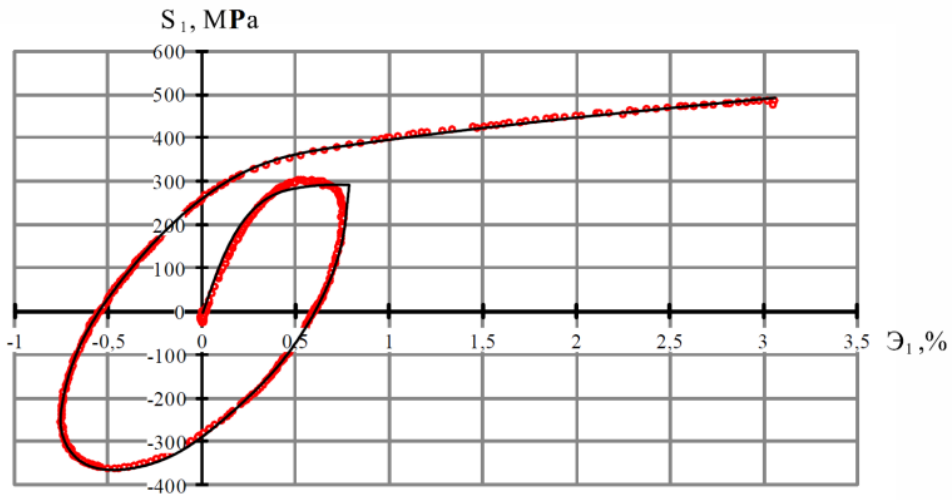

Fig. 7. Strain diagram of tension-compression of material $S_{1}-Э_{1}$

Figure 8 shows the local diagram of torsion deformation of the material $S_{3}-Э_{3}$ 


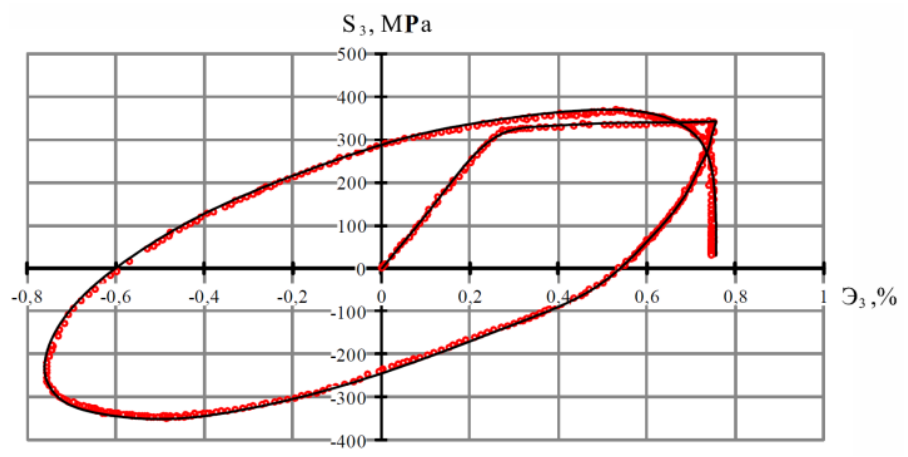

Fig. 8. Diagram of deformation of torsion of the material $S_{3}-Э_{3}$

In figure 2 - 7 experimental points are marked with circles, solid lines are marked with curves, which were built according to the considered mathematical model of the theory of processes in flat tasks, taking into account the approximation of the process functional (15) all the parameters of the complexity of the process $s, \kappa_{1}, \vartheta_{1}^{0}$ for flat trajectories and a generalized effect of baushinger. As you can see, numerical calculations on the presented mathematical model of the theory of processes using functional approximations (15) and (16) quite well correspond to the experimental data for this type of trajectory from scalar data.

\section{References}

1. A.A. Ilyushin, Plasticity. Bases of the General Mathematical Theory, Moscow: Izdatelstvo AS USSR, 271 p. (1963)

2. A.A. Ilyushin, Continuum Mechanics, Moscow: Izdatelstvo MSU, 310 p. (1990)

3. V.G. Zubchaninov, Mechanics of processes in plastic environments, Moscow: Fizmatlit, 352 p. (2010)

4. A.A. Ilyushin, Plasticity. Elastic-plastic deformation, Moscow: Gostekhizdat, 376 p. (1948)

5. V.G. Zubchaninov, A.A. Alekseev, V.I. Gultyaev, Modeling, Problems of strength and plasticity, Vol. 77, 2, pp. 113-123 (2015)

6. V.G. Zubchaninov, A.A. Alekseev, V.I. Gultyaev, Problems of strength and plasticity, Vol. 76, 1, pp. 18-25 (2017)

7. V.G. Zubchaninov, A.A. Alekseev, E.G. Alekseeva, Materials Physics and Mechanics (MPM), Vol. 24, 2, pp. 107-118 (2015)

8. V.G. Zubchaninov, Mechanics of Solids, vol. 46, 1, pp. 21-29 (2011)

9. R.A. Vasin, Mechanics of Solids, vol. 46, 1, pp. 15-20 (2011)

10. V.G. Zubchaninov, Problems of mechanics: the collection of scientific papers, Moscow: Fizmatlit, pp. 394-405 (2003)

11. V.G. Zubchaninov, A.A. Alekseev, V.I. Gultyaev, PNRPU Mechanics Bulletin, 1, pp. 94-105 (2013)

12. V.A. Golenkov, V.G.Malinin, N.A. Malinina, Structural-analytical mesomechanics and its applications, Moscow: Mashinostroenie, 634 p. (2009)

13. I.A Volkov, Iu.G Korotkikh, Equation of state viscoelastoplastic environments with damages, Moscow: Fizmatlit, 424 p. (2008). 